\title{
Real-World Single-Center Comparison of the Safety and Efficacy of Entecavir, Tenofovir Disoproxil Fumarate, and Tenofovir Alafenamide in Patients with Chronic Hepatitis B
}

\author{
Sara Jeong Hyun Phil Shin Ha Il Kim \\ Department of Gastroenterology and Hepatology, Kyung Hee University College of Medicine, Kyung Hee University \\ Hospital at Gangdong, Seoul, South Korea
}

\author{
Keywords \\ Chronic hepatitis B · Entecavir - Tenofovir - Treatment \\ outcome Safety
}

\begin{abstract}
Introduction: Chronic hepatitis B (CHB) is a major cause of chronic liver diseases and tenofovir disoproxil fumarate (TDF), tenofovir alafenamide (TAF), and entecavir (ETV) are recommended as primary treatments. This study aimed to evaluate the efficacy and safety of ETV, TDF, and TAF in a real-world clinical setting. Methods: In this retrospective cohort study, a total of $363 \mathrm{CHB}$ patients who were treated with $\operatorname{ETV}(n=163), \operatorname{TDF}(n=154)$, or TAF $(n=46)$ from July 2007 to September 2019 were enrolled. Results: Median patient age was 51 years and $66.4 \%$ of patients were male. Median duration of treatment with ETV, TDF, or TAF was 49.0 months (interquartile range, $27.0-74.0$ months). In terms of safety, cholesterol was mildly increased in the ETV and TAF groups and significantly lowered in the TDF group than baseline $(p<$ $0.001)$. There was no significant difference in liver cirrhosisrelated complications among the 3 groups at 48 weeks ( $p=$ 0.235). Hepatitis $B$ e antigen seroconversion, complete virological response, and alanine aminotransferase normaliza-
\end{abstract}

karger@karger.com www.karger.com/int

Karger $\stackrel{\text { ' }}{5}$

GOPEN ACCESS
C 2021 The Author(s)

Published by S. Karger AG, Basel

This is an Open Access article licensed under the Creative Commons Attribution-NonCommercial-4.0 International License (CC BY-NC) (http://www.karger.com/Services/OpenAccessLicense), applicable to the online version of the article only. Usage and distribution for commercial purposes requires written permission. tion at 48 weeks as measures of treatment efficacy were not significantly different among the 3 groups ( $p=0.142,0.538$, and 0.520 , respectively). There was also no significant difference in cumulative incidence rate of hepatocellular carcinoma (HCC) between the ETV and TDF groups $(p=0.894)$. Conclusions: ETV, TDF, and TAF were safe antiviral agents and showed similar antiviral effect for $\mathrm{CHB}$ at 48 weeks. Cirrhosisrelated complications and annual HCC incidence rates did not differ significantly between the ETV and TDF groups over the 48 week follow-up period.

(c) 2021 The Author(s)

Published by S. Karger AG, Basel

\section{Introduction}

Approximately 257 million people worldwide have chronic hepatitis B virus (HBV) infection. The prevalence of HBV infection is 6\% in Africa, 2\% in Southeast Asia, and $1 \%$ in the America $[1,2]$. In 2016, the Korea NationalHealth and Nutrition Examination Survey (KNHANES) estimated that the prevalence of HBV infection in Korea was about $3 \%$. Due to vaccination and prevention of perinatal HBV transmission in South Korea, the incidence of HBV has been decreasing in the 10-18 year age-group;
Correspondence to:

Hyun Phil Shin, drshp@khu.ac.kr 
the proportion of hepatitis B surface antigen positivity decreased from $2.2 \%$ in 1998 to $1.9 \%$ in 2007 and $0.3 \%$ in 2016 [3]. Without proper treatment, however, chronic hepatitis $\mathrm{B}(\mathrm{CHB})$ virus infection can cause advanced liver disease, including liver cirrhosis (LC) and hepatocellular carcinoma (HCC) [4]. As the risk of cirrhosis and its complications are associated with higher HBV DNA levels, viral suppression is very important [5]. Long-term suppression of viral replication plays an important role in slowing disease progression because complete eradication of CHB is almost impossible [6]. Lifelong treatment is generally required because hepatitis B surface antigen loss, which is considered a functional cure, is rarely achieved and $\mathrm{CHB}$ recurs in many $\mathrm{CHB}$ patients after offtherapy $[7,8]$. Effective treatments to suppress viral replication exist, but limitations such as resistance and drug safety issues arise with prolonged use $[8,9]$

Potent nucleos(t)ide analogs (NAs) with high barriers to resistance are recommended for the treatment of $\mathrm{CHB}$, such as tenofovir disoproxil fumarate (TDF), tenofovir alafenamide (TAF), and entecavir (ETV); these are currently the most widely used antiviral agents for $\mathrm{CHB}[8$, 10]. Previously, ETV and TDF were used as first-line treatment options, and then TAF was added in 2017.

ETV is not recommended in patients with lamivudine or telbivudine resistance because resistance rates to $\mathrm{rETV}$ are high in these patients [11]. Prolonged use of TDF inhibits viral activity, which in many patients lead to the regression of fibrosis and cirrhosis [12]. In addition, TDF resistance was not observed in $\mathrm{CHB}$ patients who used TDF for 6 years [13]. TDF has a good safety profile, but nephrotoxicity and osteoporosis have been reported [14, 15]. Previous studies have shown that tenofovir (TFV) toxicity is associated with a decrease in estimated glomerular filtration rate (eGFR) due to targeting of proximal tubules [16-18], and severe TFV toxicity can lead to bone disease by reducing reabsorption of phosphate by the proximal tubules, leading to phosphaturia and de-mineralization of bone [19]. TAF, a phosphonate prodrug of TFV, was developed to address some of the safety concerns associated with TFV [20], and the European Association for the Study of the Liver (EASL) recommended use of TAF as a first-line treatment for CHB in April 2017 [8]. Compared to TDF, TAF has higher plasma stability and efficiently delivers active metabolites to the liver with less systemic exposure [21]. The exposure of TFV to plasma is reduced by an average of $91 \%$ when administered in the form of TAF rather than TDF [22]. Low circulating TFV levels presumably reduce renal side effects [23]. Choi et al. [24] reported that TDF therapy decreased the risk of HCC recurrence compared with ETV treatment, [25] but comparison of the antiviral efficacy of ETV and TDF therapy is also controversial.

While a number of studies have compared ETV and TDF, [26-28] few have compared the efficacy and safety of ETV, TDF, and TAF based on real-world clinical data [29]. The main parameters used to assess clinical outcomes in $\mathrm{CHB}$ are complete virological response (CVR) and biochemical response, for example, alanine aminotransferase (ALT) normalization [30, 31]. In the current report, we investigated the safety and efficacy of ETV, TDF, and TAF in terms of changes in creatinine levels, eGFR, cholesterol, alkaline phosphatase (ALP), hepatitis $\mathrm{B}$ e antigen (HBeAg) seroconversion, CVR rate, ALT normalization, cirrhosis-related complications, and HCC incidence rate.

\section{Patients and Methods}

\section{Study Design}

This retrospective cohort study included patients who received ETV, TDF, or TAF at the Kyung Hee University Hospital at Gangdong, Seoul, South Korea. Data were collected from the electronic medical records of $701 \mathrm{CHB}$ patients who were treated with ETV, TDF, or TAF from July 2007 to September 2019.

\section{Population}

Adults over 18 years old who started taking ETV, TDF, or TAF were eligible to participate. Exclusion criteria were as follows: (1) antiviral therapy for $<48$ weeks; (2) pretreatment serum HBV DNA level of $<2,000 \mathrm{IU} / \mathrm{mL}$; (3) incomplete electronic medical records; (4) patients diagnosed with chronic kidney disease (CKD); or (5) patients being treated for HCC before enrollment.

\section{Data Collection and Definition}

Data were collected from electronic medical records at the Kyung Hee University Hospital at Gangdong and included clinical information, and laboratory data. Medical history, such as LC, hypertension, diabetes mellitus, history of taking lipid-lowering agents, tuberculosis, malignancy, chemotherapy, and immunosuppressive therapy was included in the demographic data. $\mathrm{Pa}$ tients underwent routine blood chemistry test and virological assessments, including hemoglobin, platelets, international normalized ratio of prothrombin time, liver function tests, serum HBV-DNA level, and status of HBeAg every 3-6 months. According to our outpatient protocol, patients were also followed up every 6-12 months with ultrasonography and serum alpha-fetoprotein level to screen for HCC.

We calculated eGFR using the modification of diet in renal disease GFR equation as $186 \times(\text { Serum } C r)^{-1.154} \times(\text { age })^{-0.203} \times 1.212$ (if patient was black) $\times 0.742$ (if female). HBV DNA was measured by a quantitative polymerase chain reaction assay, and CVR was defined as an HBV DNA level below $20 \mathrm{IU} / \mathrm{mL}$. Diagnosis of LC was clinically defined taking into account platelet count, serum albumin level, prothrombin time, international normalized ratio, 
Fig. 1. Flowchart of participant enrollment. $\mathrm{CHB}$, chronic hepatitis B; ETV, entecavir; TDF, tenofovir disoproxil fumarate; TAF, tenofovir alafenamide; CKD, chronic kidney disease.
CHB patients who recived ETV, TDF or TAF treatment from 2007 July to 2019 September $(n=701)$

Excluded $(n=338)$

Treated for $<48$ weeks $(n=105)$ Drug interruption or change $(n=31)$ Incomplete medical record $(n=127)$ Low level viremia at baseline $(n=62)$ Patients diagnosed with CKD $(n=13)$ and radiological image findings included splenomegaly $(>12 \mathrm{~cm})$ and liver surface nodularity. Endoscopic findings such as esophageal or gastric varices were considered in the diagnosis of LC.

Diagnosis of HCC was based on dynamic computed tomography, dynamic contrast-enhanced magnetic resonance imaging, and/or histological findings. Complications of cirrhosis included gastroesophageal varices, variceal bleeding, ascites, spontaneous bacterial peritonitis, and hepatic encephalopathy.

\section{Statistical Analysis}

Data are expressed as numbers (\%), medians (interquartile ranges), or means \pm standard deviations. The significance of differences in continuous variables between groups was assessed using Student's $t$ test or the Mann-Whitney test, while one-way ANOVA or the Kruskal-Wallis test was used to assess the significance of differences among 3 groups. Categorical variables were examined by $\chi^{2}$ test or Fisher's exact test. Cumulative incidence rates of LC-related complication and HCC were evaluated by the Kaplan-Meier method. Statistical analyses were performed using SPSS software version 24.0 (SPSS Inc., Chicago, IL, USA) and the $\mathrm{R}$ software package (R version 3.5.1, www.r-project.org). A 2-sided $p$ value $<0.05$ was considered to indicate statistical significance.

\section{Results}

\section{Population and Baseline Characteristics}

A total of 701 patients with $\mathrm{CHB}$ who were treated with ETV, TDF, or TAF were identified (Fig. 1). We excluded 338 patients who were treated with antiviral agents for $<48$ weeks $(n=105)$, had their prescription changed or stopped taking the drug $(n=31)$, had incomplete electronic medical records $(n=127)$, had low-level viremia at baseline $(n=62)$, or were diagnosed with CKD $(n=13)$. A total of 363 patients (163 patients treated with ETV, 154 patients treated with TDF, and 46 patients treated with TAF) were therefore enrolled in the study. Baseline characteristics and laboratory characteristics of these patients are shown in Table 1.

Median age of patients was 51 years and $66.4 \%$ of patients were male. There were some differences among the 3 groups. Median duration of treatment with ETV, TDF, and TAF was 75.0 months (interquartile range, 45.094.0), 43.0 months (interquartile range, 27.0-61.3), and 13.0 months (interquartile range, $12.0-13.0$ ), respectively $(p<0.001)$. There were also significant differences in the number of patients previously treated with NAs. The proportion of NA naive patients was $93.3,73.4$, and $78.2 \%$ in the ETV, TDF, and TAF groups, respectively $(p<0.001)$.

\section{Safety and Efficacy of ETV, TDF, and TAF Treatment at 48 Weeks}

To evaluate the safety of ETV, TDF, and TAF treatment at 48 weeks after administration, changes in cholesterol, ALP, creatinine, and the presence of LC-related complications were evaluated (Table 2). At 48 weeks, cholesterol level had decreased in the TDF group and mildly increased in the ETV and TAF groups compared to baseline $(p=0.000)$. eGFR changes were not signifi- 
Table 1. Baseline characteristics of the ETV, TDF, and TAF treatment groups

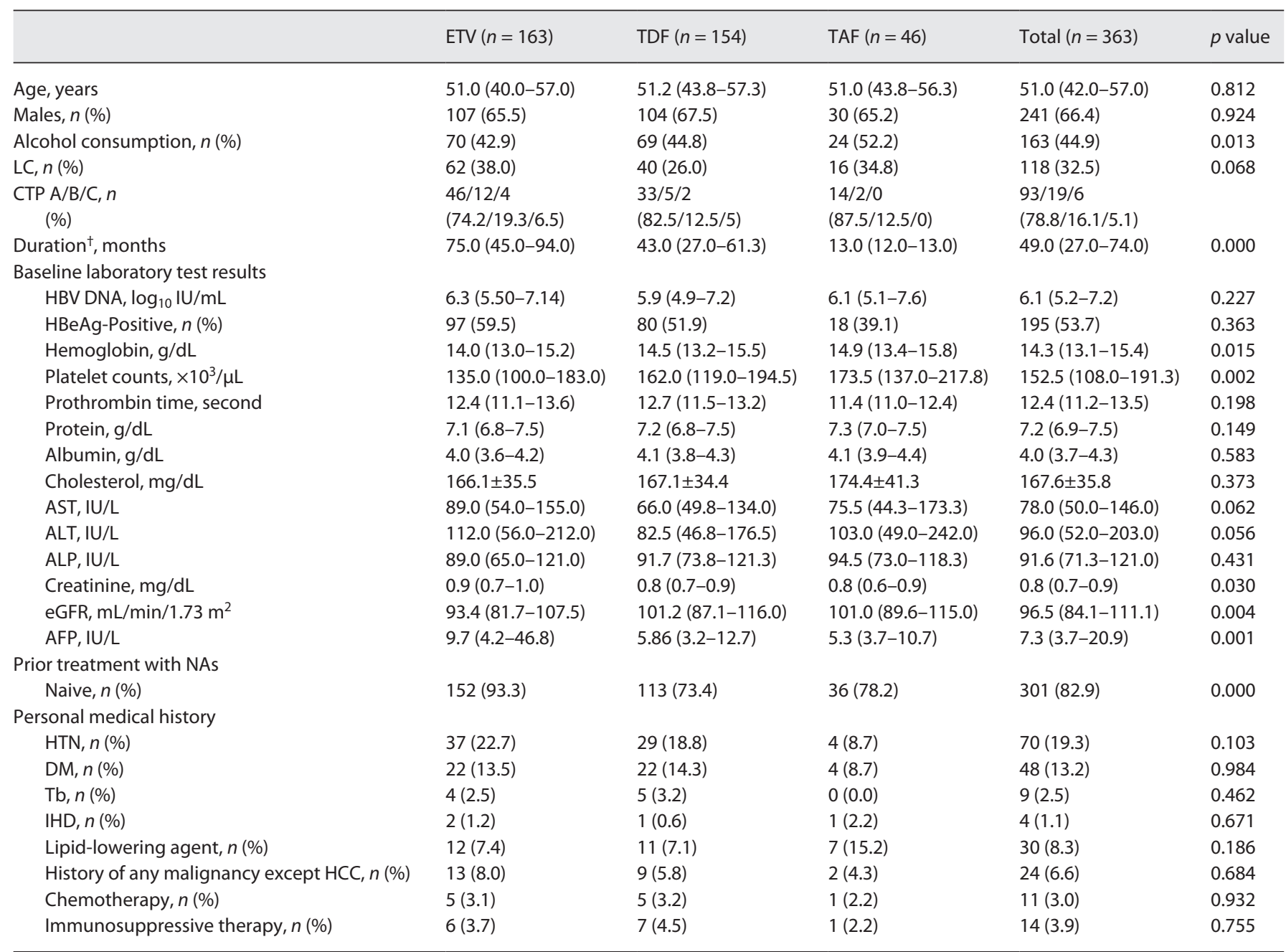

Values are expressed as numbers (\%), medians (interquartile ranges), or means \pm standard deviations. ETV, entecavir; TDF, tenofovir disoproxil fumarate; TAF, tenofovir alafenamide; LC, liver cirrhosis; CTP, Child-Turcotte-Pugh; AST, aspartate aminotransferase; ALT, alanine aminotransferase; ALP, alkaline phosphatase; eGFR, estimated glomerular filtration rate; AFP, alpha-fetoprotein; NA, nucleos(t)ide analog; HTN, hypertension; DM, diabetes mellitus; Tb, tuberculosis; IHD, ischemic heart disease; HCC, hepatocellular carcinoma; HBeAg, hepatitis B e antigen; PT, prothrombin time; HBV, hepatitis B virus; ANOVA, analysis of variance. $p<0.05$ is considered statistically significant. ${ }^{*}$ Calculated by Kruskal-Wallis test (or one-way ANOVA test, if appropriate) and $x^{2}$ test (or Fisher's exact test, if appropriate). + Duration of current NA medication.

cantly different between the 3 groups $(p=0.134)$ at 48 weeks, nor were ALP changes $(p=0.826)$. There was no significant difference in LC complications after 48 weeks among groups $(p=0.235)$. In the ETV group, 3 patients developed LC complications, 2 developed variceal bleeding, and 1 patient developed ascites. There was only 1 case of variceal bleeding in the TDF group and no LC complications after 48 weeks in the TAF group.

HBeAg seroconversion, CVR, and ALT normalization were used to evaluate the efficacy of ETV, TDF, and TAF (Table 2). The number of patients who were HBeAg pos- itive at the start of the study was 97 of 163 in the ETV group, 80 of 154 in the TDF group, and 18 of 46 in the TAF group. The HBeAg seroconversion rates in the ETV, TDF, and TAF groups were not significantly different at $26.8,15.0$, and $16.7 \%(p=0.142)$. A total of 128 of the 163 ETV-treated patients (78.5\%), 127 of the 154 TDF-treated patients $(82.5 \%)$, and 35 of the 46 TAF-treated patients (76.1\%) achieved CVR $(p=0.538)$. ALT normalization was similar among groups; $82.1,77.0$, and $78.3 \%$ of patients in the ETV, TDF, and TAF group, respectively $(p=$ 0.520 ). 
Table 2. Comparison of safety and efficacy in laboratory parameters and LC-related complications between baseline and 48 weeks in the ETV, TDF, and TAF groups

\begin{tabular}{|c|c|c|c|c|c|}
\hline & $\operatorname{ETV}(n=163)$ & $\operatorname{TDF}(n=154)$ & $\operatorname{TAF}(n=46)$ & Total $(n=363)$ & $p$ value \\
\hline$\Delta$ Cholesterol (mg/dL) & $6(-11 \sim 22)$ & $-10(-28.0 \sim 8)$ & $5.50(-22.25 \sim 30.75)$ & $0.00(-23.00-18.00)$ & $0.000^{*}$ \\
\hline$\triangle \mathrm{ALP}, \mathrm{IU} / \mathrm{L}$ & $-6.00(-28.33 \sim 18.67)$ & $-5.50(-4.25 \sim 28.00)$ & $-11.00(-20.00 \sim 8.00)$ & $-6.67(-31.00 \sim 18.70)$ & 0.826 \\
\hline$\Delta \mathrm{eGFR}, \mathrm{mg} / \mathrm{dL}$ & $-0.40(-12.20 \sim 6.70)$ & $-3.35(-16.13 \sim 10.70)$ & $1.90(-6.73 \sim 15.19)$ & $-0.50(-12.3 \sim 8.60)$ & 0.134 \\
\hline$\Delta \mathrm{eGFR} \geq 30 \%, n(\%)$ & $7(4.3)$ & $4(2.6)$ & $0(0)$ & $11(3.0)$ & 0.393 \\
\hline Complications of LC, $n(\%)$ & $3(1.8)$ & $1(0.7)$ & $0(0)$ & $4(1.1)$ & 0.235 \\
\hline Varices & $0(0)$ & $0(0)$ & $0(0)$ & $0(0)$ & \\
\hline Variceal bleeding & $2(66.7)$ & $1(100)$ & $0(0)$ & $3(75)$ & \\
\hline Ascites & $1(33.3)$ & $0(0)$ & $0(0)$ & $1(25)$ & \\
\hline SBP & $0(0)$ & $0(0)$ & $0(0)$ & $0(0)$ & \\
\hline $\mathrm{HE}$ & $0(0)$ & $0(0)$ & $0(0)$ & $0(0)$ & \\
\hline HBeAg seroconversion, $n(\%)$ & $28 / 97(26.8)$ & $12 / 80(15.0)$ & $3 / 18(16.7)$ & $41 / 195(21.0)$ & 0.142 \\
\hline CVR, $n(\%)$ & $128(78.5)$ & $127(82.5)$ & $35(76.1)$ & $288(79.3)$ & 0.538 \\
\hline ALT normalization, $n(\%)$ & $133(82.1)$ & $117(77.0)$ & $36(78.3)$ & $286(79.4)$ & 0.520 \\
\hline
\end{tabular}

ETV, entecavir; TDF, tenofovir disoproxil fumarate; TAF, tenofovir alafenamide; ALP, alkaline phosphatase; eGFR, estimated glomerular filtration rate; LC, liver cirrhosis; SBP, spontaneous bacterial peritonitis; HE, hepatic encephalopathy; CVR, complete viral response; ALT, alanine aminotransferase; HBeAg, hepatitis B e antigen. $p<0.05$ is considered statistically significant.

Table 3. Safety and efficacy of ETV and TDF at 48 weeks

\begin{tabular}{|c|c|c|c|c|}
\hline & $\operatorname{ETV}(n=163)$ & $\operatorname{TDF}(n=154)$ & Total $(n=317)$ & $p$ value \\
\hline$\Delta$ Cholesterol, mg/dL & $6(-11 \sim 22)$ & $-10(-28.0 \sim 8)$ & $0.00(-23.00 \sim 16.00)$ & $0.000^{*}$ \\
\hline$\triangle \mathrm{ALP}, \mathrm{IU} / \mathrm{L}$ & $-6.00(-28.33 \sim 18.67)$ & $-5.50(-4.25 \sim 28.00)$ & $-6.00(-31.83 \sim 23.33)$ & 0.580 \\
\hline Complications of LC, $n(\%)$ & $3(1.8)$ & $1(0.7)$ & $4(1.1)$ & 0.114 \\
\hline Varices & $0(0)$ & $0(0)$ & $0(0)$ & \\
\hline Variceal bleeding & $2(66.7)$ & $1(100)$ & $3(75)$ & \\
\hline HE & $0(0)$ & $0(0)$ & $0(0)$ & \\
\hline HBeAg seroconversion & 28/97 (26.8\%) & $12 / 80(15.0 \%)$ & $38 / 177$ (21.5\%) & 0.057 \\
\hline CVR & $128 / 163(78.5 \%)$ & $127 / 154(82.5 \%)$ & $255 / 317(80.4 \%)$ & 0.377 \\
\hline ALT normalization & $133 / 162(82.1 \%)$ & 152/117 (77.0\%) & $250 / 314(79.6 \%)$ & 0.260 \\
\hline
\end{tabular}

ETV, entecavir; TDF, tenofovir disoproxil fumarate; ALP, alkaline phosphatase; eGFR, estimated glomerular filtration rate; LC, liver cirrhosis; SBP, spontaneous bacterial peritonitis; HE, hepatic encephalopathy; CVR, complete viral response; ALT, alanine aminotransferase; $\mathrm{HBeAg}$, hepatitis B e antigen. $p<0.05$ is considered statistically significant.

Safety and Efficacy of ETV and TDF at 48 Weeks

At 48 weeks after ETV or TDF administration, cholesterol increased by $6 \mathrm{mg} / \mathrm{dL}$ in the ETV group and decreased by $10 \mathrm{mg} / \mathrm{dL}$ in the TDF group ( $p=0.000$ ). However, there were no significant differences in changes in ALP, eGFR, or LC complications between these 2 groups (Table 3). Differences in HBeAg seroconversion, CVR, and ALT normalization were not significantly different between the ETV and TDF groups, indicating that these 2 drugs had similar efficacy (Table 3 ).
Safety and Efficacy of ETV and TAF at 48 Weeks

Table 4 shows the results of laboratory parameters used to assess the safety of ETV and TAF. There were no differences between these 2 groups in cholesterol, ALP, or creatinine levels, or the incidence of LC complications. In terms of efficacy, there were no differences in $\mathrm{HBeAg}$ seroconversion, CVR, or ALT normalization between the 2 groups ( $p=0.363,0.724,0.556$, respectively, Table 4). 
Table 4. Safety and efficacy of ETV and TAF at 48 weeks

\begin{tabular}{llll}
\hline & ETV $(n=163)$ & TAF $(n=46)$ & Total $(n=209)$ \\
\hline$\Delta$ Cholesterol, mg/dL & $6(-11 \sim 22)$ & $5.50(-22.25 \sim 30.75)$ & $6.00(-13.00 \sim 24.50)$ \\
$\Delta$ ALP, IU/L & $-6.00(-28.33 \sim 18.67)$ & $-11.00(-20.00 \sim 7.25)$ & $-8.33(-26.50 \sim 13.33)$ \\
$\Delta$ eGFR, mg/dL & $-0.40(-12.20 \sim 6.70)$ & $1.90(-6.73 \sim 15.19)$ & $-0.40(-10.75 \sim 7.70)$ \\
Complications of LC, $n(\%)$ & $3(1.8)$ & $0(0)$ & $3(1.8)$ \\
$\quad$ Varices & $0(0)$ & $0(0)$ & $0(0)$ \\
$\quad$ Variceal bleeding & $2(66.7)$ & $0(0)$ & $2(66.7)$ \\
$\quad 1(33.3)$ & $0(0)$ & $1(33.3)$ \\
$\quad$ Ascites & $0(0)$ & $0(0)$ & $0(0)$ \\
$\quad$ HE & $0(0)$ & $0(0)$ & 0.969 \\
HBeAg seroconversion & $28 / 97(26.8 \%)$ & $3 / 18(16.7 \%)$ & $29 / 115(25.5 \%)$ \\
CVR & $128 / 163(78.5 \%)$ & $35 / 46(76.1 \%)$ & $161 / 209(78.0 \%)$ \\
ALT normalization & $133 / 162(82.1 \%)$ & $36 / 46(78.3 \%)$ & $169 / 208(81.3 \%)$ \\
\hline
\end{tabular}

ETV, entecavir; TAF, tenofovir alafenamide; ALP, alkaline phosphatase; eGFR, estimated glomerular filtration rate; LC, liver cirrhosis; SBP, spontaneous bacterial peritonitis; $\mathrm{HE}$, hepatic encephalopathy; CVR, complete viral response; $\mathrm{ALT}$, alanine aminotransferase; $\mathrm{HBeAg}$, hepatitis $B$ e antigen. $p<0.05$ is considered statistically significant.

Table 5. Safety and efficacy of TAF and TDF at 48 weeks

\begin{tabular}{|c|c|c|c|c|}
\hline$\Delta$ Cholesterol, $\mathrm{mg} / \mathrm{dL}$ & $-10.00(-28.00 \sim 8.00)$ & $5.50(-22.25 \sim 30.75)$ & $-8.50(-26.00 \sim 12.00)$ & $0.010^{*}$ \\
\hline$\triangle \mathrm{ALP}, \mathrm{IU} / \mathrm{L}$ & $-5.50(-4.25 \sim 28.00)$ & $-11.00(-20.00 \sim 8.00)$ & $-6.83(-34.75 \sim 19.25)$ & 0.744 \\
\hline$\Delta \mathrm{eGFR}, \mathrm{mg} / \mathrm{dL}$ & $-3.35(-16.13 \sim 10.70)$ & $1.90(-6.72 \sim 8.50)$ & $-0.60(-12.50 \sim 10.38)$ & 0.071 \\
\hline Complications of LC, $n(\%)$ & $1(0.7)$ & $0(0)$ & $1(0.7)$ & 0.584 \\
\hline Varices & $0(0)$ & $0(0)$ & $0(0)$ & \\
\hline Variceal bleeding & $1(100)$ & $0(0)$ & $1(100)$ & \\
\hline SBP & $0(0)$ & $0(0)$ & $0(0)$ & \\
\hline $\mathrm{HE}$ & $0(0)$ & $0(0)$ & $0(0)$ & \\
\hline HBeAg seroconversion & $12 / 80(15.0 \%)$ & $3 / 18(16.7 \%)$ & $15 / 98(15.3 \%)$ & 0.859 \\
\hline CVR & $127 / 154(82.5 \%)$ & $35 / 46(76.1 \%)$ & $162 / 200(81.0 \%)$ & 0.333 \\
\hline ALT normalization & $117 / 152(77.0 \%)$ & $36 / 46(78.3 \%)$ & $153 / 198(77.3 \%)$ & 0.855 \\
\hline
\end{tabular}

TDF, tenofovir disoproxil fumarate; TAF, tenofovir alafenamide; ALP, alkaline phosphatase; eGFR, estimated glomerular filtration rate; LC, liver cirrhosis; SBP, spontaneous bacterial peritonitis; $H E$, hepatic encephalopathy; CVR, complete viral response; ALT, alanine aminotransferase; $\mathrm{HBeAg}$, hepatitis $\mathrm{B}$ e antigen. $p<0.05$ is considered statistically significant.

Safety and Efficacy of TAF and TDF at 48 Weeks

Cholesterol decreased by $10 \mathrm{mg} / \mathrm{dL}$ in the TDF group and increased by $5.50 \mathrm{mg} / \mathrm{dL}$ in TAF group from baseline, which showed a significant difference between the 2 groups ( $p=0.010$, Table 5 ). Table 5 shows the efficacy of TAF and TDF in CHB patients at 48 weeks. There were no differences between the 2 groups in $\mathrm{HBeAg}$ seroconversion, CVR, or ALT normalization ( $p=0.859,0.333$, 0.855 , respectively).

Safety and Efficacy of Entecavir and Tenofovir Prodrugs
Incidence of Significant Renal Impairment in the ETV, TDF, and TAF Groups at 48 Weeks

We defined significant renal impairment as more than $30 \%$ reduction in eGFR at endpoint compared to baseline. $4.3 \%(7 / 163)$ of patients in the ETV group, $2.6 \%$ $(4 / 154)$ in the TDF group, and $0 \%(0 / 46)$ in the TAF group had an eGFR reduction of more than $30 \%$. These differences in significant renal impairment were not statistically significant (Table 2, $p=0.393$ ). 


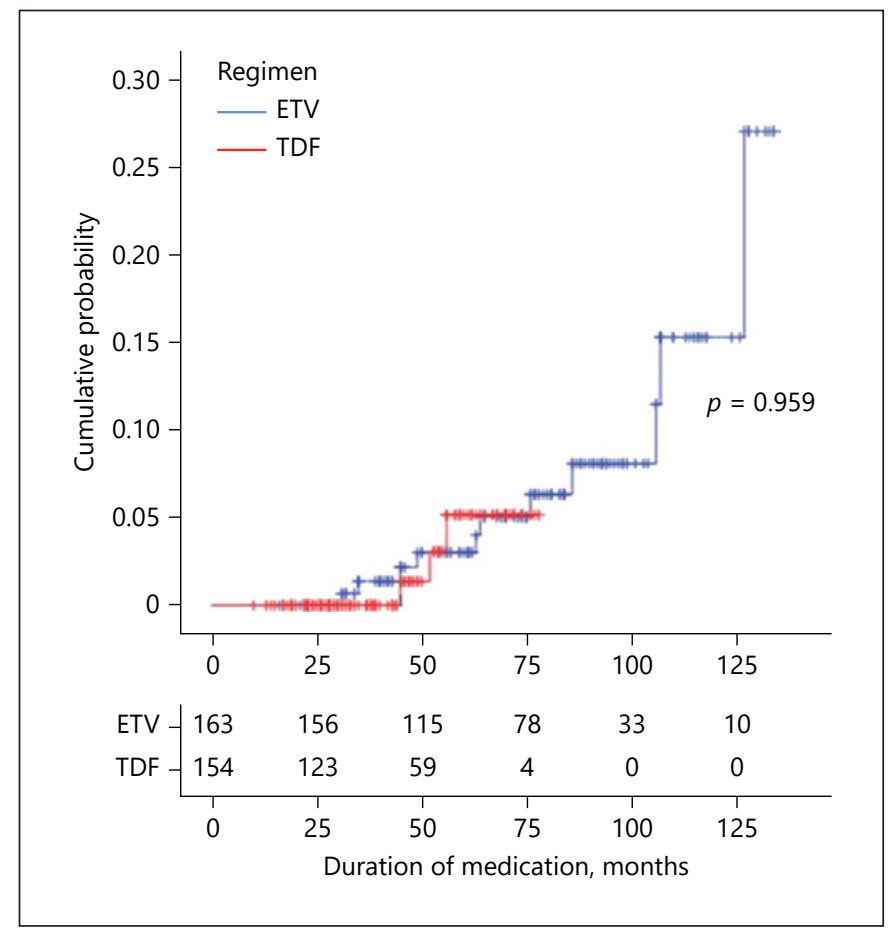

Fig. 2. Cumulative incidence rates of complications associated with liver cirrhosis. ETV, entecavir; TDF, tenofovir disoproxil fumarate.

\section{Comparison of LC Complications between the ETV and TDF Groups}

Cumulative incidence of LC-related complication requiring hospitalization is shown in Figure 2. Because the median duration of treatment in the TAF-treated group was 13.0 months, cumulative incidence, and annual incidence were analyzed only in the ETV and TDF groups. Annual incidence rate of LC-related complication was 0.601 per 100-person years in the ETV group and 0.299 per 100-person years in the TDF group, which was not a significant difference (Fig. 2, $p=0.959$ ).

During the follow-up period, 11 patients in the ETV group needed hospitalization due to LC-related complications; 3 due to varices, 3 due to variceal bleeding, 3 due to ascites, 1 due to spontaneous bacterial peritonitis, and 1 due to hepatic encephalopathy. Three patients in the TDF group were hospitalized due to complications: 2 with variceal bleeding and 1 with hepatic encephalopathy.

\section{Incidence Rates of HCC in the ETV and TDF Groups}

Figure 3 shows the cumulative incidence rate of HCC. Like LC-related complications, the cumulative incidence and annual incidence of HCC were analyzed only in the

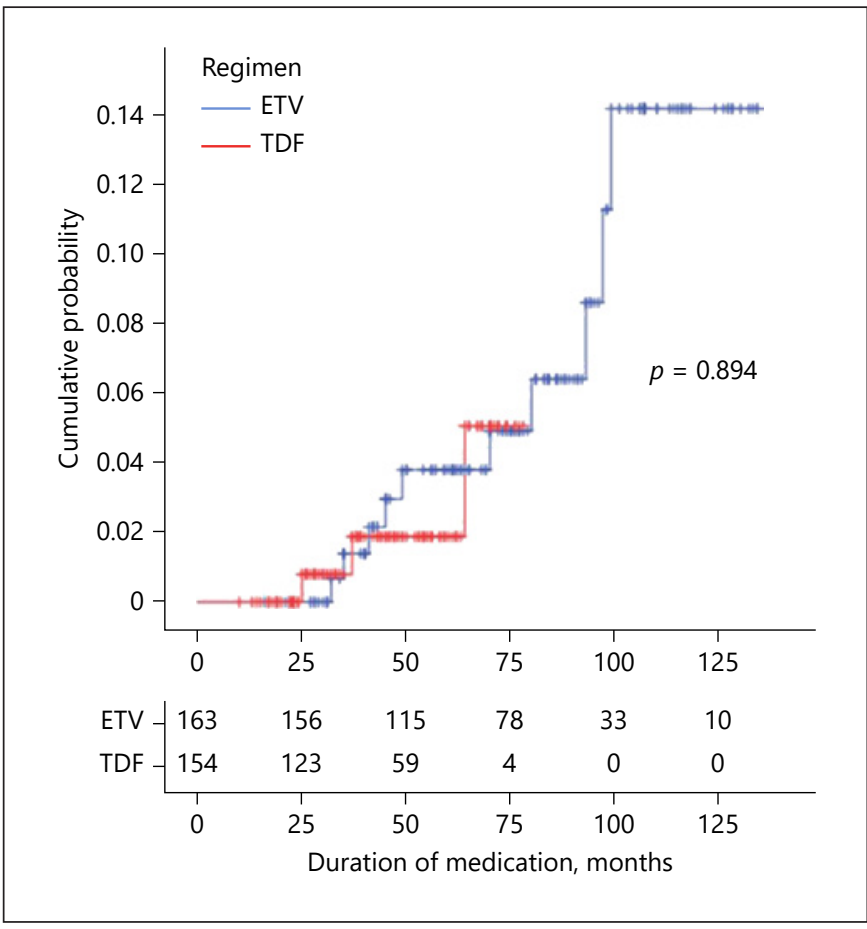

Fig. 3. Cumulative incidence rates of hepatocellular carcinoma. ETV, entecavir; TDF, tenofovir disoproxil fumarate.

ETV and TDF groups due to the short duration of TAF treatment. Annual incidence rate of HCC was 0.552 per 100 -person years in the ETV group and 0.299 per 100 -person years in the TDF group. This difference between groups was not significant (Fig. 3, $p=0.894$ ).

HCC occurred in 10 of the 163 ETV-treated patients (6.13\%), 3 of the 154 TDF-treated patients (1.95\%), and no TAF-treated patient during the follow-up period. However, these differences among groups were not statistically significant $(p=0.230)$.

\section{Discussion}

We analyzed the safety and efficacy of ETV, TDF, and TAF for CHB treatment based on real-world, single-center data. ETV, TDF, and TAF are first-line therapies for CHB in the EASL guidelines and AASLD guidance $[8,11]$. A number of studies have compared ETV and TDF, but there is a paucity of real-world clinical data to compare patients treated with ETV, TDF, or TAF. In this study, we compared the safety and efficacy of ETV, TDF, and TAF and evaluated significant renal impairment. 
A total of 701 patients were initially selected for this study; after exclusion of 338 patients, data from 363 were analyzed. In safety analysis of a drug, all patients who took that medication should be included so that patients who discontinue treatment after a short period due to side effects are not excluded. However, a number of patients who took the medication for a short period were excluded from our study based on our exclusion criteria, which is a limitation of this study.

A total of 13 patients were excluded due to underlying $\mathrm{CKD}$. The reason for excluding CKD patients is that ETV and TDF, unlike TAF, require dose reduction in $\mathrm{CKD}$, complicating comparison of safety and efficacy at 48 weeks. In addition, eGFR was included in the evaluation of safety, and this is known to be altered in CKD.

This study also included $3 \%$ of patients with a cancer other than HCC at the time of drug initiation. The safety of the drugs in these patients could not be analyzed because of the small number of patients.

After 48 weeks of administration, total cholesterol level decreased significantly in the TDF-treated group compared to baseline. The lipid-lowering effect of TDF has been noted in several articles, but the underlying mechanism has not been clarified [32-35]. In our study, total cholesterol level increased in the TAF-treated group. This is consistent with previous studies that reported that TAF increases cholesterol levels $[36,37]$.

Severe TDF toxicity can lead to bone disease, such as osteoporosis [19]. But Wei et al. [38] reported that there is no significant increase in the incidence of osteopenia/ osteoporosis in patients treated with TDF or ETV. We measured ALP to assess the bone toxicity of ETV, TDF, and TAF. In all 3 groups, ALP levels decreased from baseline at 48 weeks, as did other liver function test parameters, with no significant differences among treatment groups. To more accurately evaluate osteoporosis, serum calcium, phosphate, and bone mineral density (BMD) are required. We did not evaluate BMD before and after drug administration in our study, which is a limitation of our study. However, bone resorption could cause compensatory osteoblast activation as indicated by increased serum ALP, and a significant association between TDF use and increased serum ALP has been reported by several research groups [39-42].

To evaluate renal toxicity, eGFR was compared at baseline and after 48 weeks, and there were no significant differences among the 3 groups in terms of changes in eGFR $(p=0.134)$. Trinh et al. [43] reported changes in renal function in patients with $\mathrm{CHB}$ treated with TDF $(n=239)$ or ETV ( $n=171)$. In Trinh et al. [43] study, TDF did not

Safety and Efficacy of Entecavir and Tenofovir Prodrugs appear to worsen renal function to a greater extent than ETV during the follow-up period among patients without significant renal impairment, but in patients with renal dysfunction, eGFR decreased to a greater extent in the TDF-treated group than the ETV group. Our study showed similar result, but it was conducted only in patients without CKD. Additional studies that include CKD patients are required to verify our findings. TAF or ETV should be considered in patients who are at risk for renal dysfunction [8]. In this study, eGFR decreased to a greater extent in the ETV group than the TAF group but without statistical significance $(p=0.055)$. Although we wanted to compare changes in renal function among the ETV, TDF, and TAF groups after 48 weeks of follow-up, the average follow-up period in the TAF group was only 13 months. Future studies should include the cumulative probability of renal toxicity during the total follow-up period.

To evaluate renal safety, we evaluated the number of patients with significant renal impairment after 48 weeks. According to the RIFLE criteria, an eGFR increase of more than $25 \%$ predisposes to acute kidney injury [44]. We defined significant renal impairment as a more than $30 \%$ reduction in eGFR at endpoint compared to baseline. There was no significant difference in the number of patients with significant renal impairment at 48 weeks among the 3 groups $(p=0.393)$. We also analyzed $\mathrm{HBeAg}$ seroconversion, CVR, and ALT normalization to evaluate the efficacy of ETV, TDF, and TAF and observed no significant differences in these parameters among the 3 groups at 48 weeks, consistent with the previous studies $[29,45,46]$

CVR at 48 weeks was $78.5 \%$ in the ETV group, $82.5 \%$ in the TDF group, and $76.1 \%$ in the TAF group. In future studies, cumulative incidence and annual incidence of CVR should be compared among the 3 groups; we were unable to do this because of the short treatment duration with TAF. Detailed analysis according to the pretreatment state was not done. Choi et al. [25] study [25, 47] suggested that TFV treatment was associated with lower risk of HCC than ETV, but another study found no difference between the 2 drugs [48].

Because the median duration of TAF treatment was 13 months in the current study, we analyzed the cumulative incidence and annual incidence of LC-related complications and HCC only in the ETV and TDF groups. There were no differences in the cumulative incidence or annual incidence of LC complications and HCC between these 2 groups, but the fact that the observation period was different between the ETV and TDF groups is a limitation of our study. 
Other limitations are as follows. First, this study was conducted in a single-center, with a small number of patients, especially in the TAF treatment group. TAF has been used as a first-line treatment for CHB since 2017, and it started being prescribed to patients at our hospital in December 2017. Therefore, finding patients who took TAF for more than 48 weeks was challenging. Second, the observation periods and baseline characteristics were different between the 3 treatment groups in this retrospective study. Because the TAF-treated group has a significantly shorter follow-up period than other 2 groups, CVR, cirrhosis-related complications, and cumulative incidence over a 48 week period could not be compared among all 3 groups. Third, when comparing safety, no BMD tests were available to determine the occurrence of osteoporosis. Patients with impaired renal function were not included because there were insufficient data on underlying renal disease. A fourth limitation of this study is that the cumulative incidence and annual incidence of LC complications and HCC could not be compared between the TAF group and the ETV and TDF groups.

\section{Conclusions}

ETV, TDF, and TAF are similarly safe and effective antiviral agents for $\mathrm{CHB}$ based on analysis of data from a 48-week follow-up period. Cirrhosis-related complications and annual HCC incidence rates were not significantly different between the ETV- and TDF-treated groups during the 48-week follow-up period.

\section{Statement of Ethics}

This study was a retrospective analysis of existing administrative and clinical data, so informed consent was not required. This study was approved by the Institutional Review Board of the Kyung Hee University Hospital at Gangdong (IRB File No. 201911-010).

\section{Conflict of Interest Statement}

The authors declare that there are no conflicts of interest.

\section{Funding Sources}

This work received no specific grant from any funding agency in the public, commercial, or not-for-profit sectors.

\section{Author Contributions}

S. Jeong and H.P. Shin were involved in the basic structure, study design, interpretation of data, drafting of the manuscript, study supervision, and statistical analysis. H.I. Kim was involved in manuscript format and structural change.

\section{Data Availability Statement}

The data that support our findings are available on request from the corresponding author, H.P.S. They are not publicly available due to containing patients ID, gender, age, treatment regimen and final diagnosis.

\section{References}

1 Schweitzer A, Horn J, Mikolajczyk RT, Krause G, Ott JJ. Estimations of worldwide prevalence of chronic hepatitis B virus infection: a systematic review of data published between 1965 and 2013. Lancet. 2015 Oct 17; 386(10003): 1546-55.

2 WHO. Global Hepatitis Report 2017. Geneva: World Health Organization; 2017.

3 Prevention. KCfDC. Korea National Health and Nutrition Examination Survey (KNHANES) 2016. 2016.

4 Ganem D, Prince AM. Hepatitis B virus infection--natural history and clinical consequences. N Engl J Med. 2004 Mar 11;350(11): 1118-29.

5 Yuen MF, Tanaka Y, Fong DY, Fung J, Wong DK, Yuen JC, et al. Independent risk factors and predictive score for the development of hepatocellular carcinoma in chronic hepatitis B. J Hepatol. 2009 Jan;50(1):808.
6 Moon JC, Kim SH, Kim IH, Lee CH, Kim SW, Lee SO, et al. Disease progression in chronic hepatitis B patients under long-term antiviral therapy. Gut Liver. 2015 May 23;9(3):395404.

7 Terrault NA, Bzowej NH, Chang KM, Hwang JP, Jonas MM, Murad MH, et al. AASLD guidelines for treatment of chronic hepatitis B. Hepatology. 2016 Jan;63(1):261-83.

8 European Association for the Study of the Liver. EASL 2017 clinical practice guidelines on the management of hepatitis B virus infection. J Hepatol. 2017 Aug;67(2):370-98.

9 Liaw YF, Kao JH, Piratvisuth T, Chan HL, Chien RN, Liu CJ, et al. Asian-pacific consensus statement on the management of chronic hepatitis B: a 2012 update. Hepatol Int. 2012 Jun;6(3):531-61.

10 Han Y, Zeng A, Liao H, Liu Y, Chen Y, Ding $\mathrm{H}$. The efficacy and safety comparison between tenofovir and entecavir in treatment of chronic hepatitis B and HBV related cirrhosis: a systematic review and Meta-analysis. Int Immunopharmacol. 2017 Jan;42:168-75.

11 Terrault NA, Lok ASF, McMahon BJ, Chang KM, Hwang JP, Jonas MM, et al. Update on prevention, diagnosis, and treatment of chronic hepatitis B: AASLD 2018 hepatitis B guidance. Hepatology. 2018 Apr;67(4):156099.

12 Marcellin P, Gane E, Buti M, Afdhal N, Sievert W, Jacobson IM, et al. Regression of cirrhosis during treatment with tenofovir disoproxil fumarate for chronic hepatitis B: a 5-year open-label follow-up study. Lancet. $2013 \mathrm{Feb}$ 9;381(9865):468-75.

13 Kitrinos KM, Corsa A, Liu Y, Flaherty J, Snow-Lampart A, Marcellin P, et al. No detectable resistance to tenofovir disoproxil fumarate after 6 years of therapy in patients with chronic hepatitis B. Hepatology. 2014 Feb; 59(2):434-42. 
14 Gara N, Zhao X, Collins MT, Chong WH, Kleiner DE, Jake Liang T, et al. Renal tubular dysfunction during long-term adefovir or tenofovir therapy in chronic hepatitis B. Aliment Pharmacol Ther. 2012 Jun; 35(11): 1317-25.

15 Hall AM. Update on tenofovir toxicity in the kidney. Pediatr Nephrol. 2013 Jul;28(7): 1011-23.

16 Cooper RD, Wiebe N, Smith N, Keiser P, Naicker S, Tonelli M. Systematic review and meta-analysis: renal safety of tenofovir disoproxil fumarate in HIV-infected patients. Clin Infect Dis. 2010 Sep 1;51(5):496-505.

17 Jao J, Wyatt CM. Antiretroviral medications: adverse effects on the kidney. Adv Chronic Kidney Dis. 2010 Jan;17(1):72-82.

18 Perazella MA. Tenofovir-induced kidney disease: an acquired renal tubular mitochondriopathy. Kidney Int. 2010 Dec;78(11):1060-3.

19 Woodward CL, Hall AM, Williams IG, Madge S, Copas A, Nair D, et al. Tenofovir-associated renal and bone toxicity. HIV Med. 2009 Sep; 10(8):482-7.

20 Agarwal K, Fung SK, Nguyen TT, Cheng W, Sicard E, Ryder SD, et al. Twenty-eight day safety, antiviral activity, and pharmacokinetics of tenofovir alafenamide for treatment of chronic hepatitis B infection. J Hepatol. 2015 Mar;62(3):533-40.

21 Murakami E, Wang T, Park Y, Hao J, Lepist EI, Babusis D, et al. Implications of efficient hepatic delivery by tenofovir alafenamide (GS-7340) for hepatitis B virus therapy. Antimicrob Agents Chemother. 2015;59(6):35639.

22 Sax PE, Zolopa A, Brar I, Elion R, Ortiz R, Post $\mathrm{F}$, et al. Tenofovir alafenamide vs. tenofovir disoproxil fumarate in single tablet regimens for initial HIV-1 therapy: a randomized phase 2 study. J Acquir Immune Defic Syndr. 2014 Sep 1;67(1):52-8

23 DeJesus E, Haas B, Segal-Maurer S, Ramgopal MN, Mills A, Margot N, et al. Superior efficacy and improved renal and bone safety after switching from a tenofovir disoproxil fumarate- to a tenofovir alafenamide-based regimen through 96 weeks of treatment. AIDS Res Hum Retroviruses. 2018 Apr;34(4):33742.

24 Lee SW, Choi J, Kim SU, Lim YS. Entecavir versus tenofovir in patients with chronic hepatitis B: enemies or partners in the prevention of hepatocellular carcinoma. Clin Mol Hepatol. 2021 Jul;27(3):402-12.

25 Choi J, Jo C, Lim YS. Tenofovir versus entecavir on recurrence of hepatitis B virus-related hepatocellular carcinoma after surgical resection. Hepatology. 2021 Feb;73(2):661-73.

26 Woo G, Tomlinson G, Nishikawa Y, Kowgier M, Sherman M, Wong DK, et al. Tenofovir and entecavir are the most effective antiviral agents for chronic hepatitis B: a systematic review and Bayesian meta-analyses. Gastroenterology. 2010 Oct;139(4):1218-29.
27 Sriprayoon T, Mahidol C, Ungtrakul T, Chun-On P, Soonklang K, Pongpun W, et al. Efficacy and safety of entecavir versus tenofovir treatment in chronic hepatitis B patients: a randomized controlled trial. Hepatol Res. 2017 Mar;47(3):E161-8.

28 Cai D, Pan C, Yu W, Dang S, Li J, Wu S, et al. Comparison of the long-term efficacy of tenofovir and entecavir in nucleos $(\mathrm{t})$ ide analoguenaive $\mathrm{HBeAg}$-positive patients with chronic hepatitis B: a large, multicentre, randomized controlled trials. Medicine. 2019 Jan;98(1): e13983.

29 Buti M, Riveiro-Barciela M, Esteban R. Longterm safety and efficacy of nucleo(t)side analogue therapy in hepatitis B. Liver Int. 2018 Feb;38 Suppl 1(Suppl 1):84-9.

30 Jang JW, Choi JY, Kim YS, Yoo JJ, Woo HY, Choi SK, et al. Effects of virologic response to treatment on short- and long-term outcomes of patients with chronic hepatitis B virus infection and decompensated cirrhosis. Clin Gastroenterol Hepatol. 2018 Dec; 16(12): 1954-63.e3

31 Wong GL, Chan HL, Tse YK, Yip TC, Lam KL, Lui GC, et al. Normal on-treatment ALT during antiviral treatment is associated with a lower risk of hepatic events in patients with chronic hepatitis B. J Hepatol. 2018 Oct;69(4): 793-802.

32 Tungsiripat M, Kitch D, Glesby MJ, Gupta SK, Mellors JW, Moran L, et al. A pilot study to determine the impact on dyslipidemia of adding tenofovir to stable background antiretroviral therapy: ACTG 5206. AIDS. 2010 Jul 17;24(11):1781-4.

33 Valantin MA, Bittar R, de Truchis P, Bollens D, Slama L, Giral P, et al. Switching the nucleoside reverse transcriptase inhibitor backbone to tenofovir disoproxil fumarate $+\mathrm{em}$ tricitabine promptly improves triglycerides and low-density lipoprotein cholesterol in dyslipidaemic patients. J Antimicrob Chemother. 2010 Mar;65(3):556-61.

34 Fabbiani M, Bracciale L, Doino M, Sidella L, Farina S, Di Cristo V, et al. Lipid-lowering effect of tenofovir in HIV-infected patients. J Antimicrob Chemother. 2011 Mar;66(3): 682-3.

35 Santos JR, Saumoy M, Curran A, Bravo I, Llibre JM, Navarro J, et al. The lipid-lowering effect of tenofovir/emtricitabine: a randomized, crossover, double-blind, placebo-controlled trial. Clin Infect Dis. 2015 Aug 1;61(3): 403-8.

36 Cid-Silva P, Fernández-Bargiela N, Margusino-Framiñán $\mathrm{L}$, Balboa-Barreiro $\mathrm{V}$, MenaDe-Cea Á, López-Calvo S, et al. Treatment with tenofovir alafenamide fumarate worsens the lipid profile of HIV-infected patients versus treatment with tenofovir disoproxil fumarate, each coformulated with elvitegravir, cobicistat, and emtricitabine. Basic Clin Pharmacol Toxicol. 2019 Apr;124(4):479-90.
37 Kauppinen KJ, Kivelä P, Sutinen J. Switching from tenofovir disoproxil fumarate to tenofovir alafenamide significantly worsens the lipid profile in a real-world setting. AIDS Patient Care STDS. 2019 Dec;33(12):500-6.

38 Wei MT, Le AK, Chang MS, Hsu H, Nguyen $\mathrm{P}$, Zhang JQ, et al. Antiviral therapy and the development of osteopenia/osteoporosis among Asians with chronic hepatitis B. J Med Virol. 2019 Jul;91(7):1288-94.

39 Fux CA, Rauch A, Simcock M, Bucher HC, Hirschel B, Opravil M, et al. Tenofovir use is associated with an increase in serum alkaline phosphatase in the Swiss HIV Cohort Study. Antivir Ther. 2008;13(8):1077-82.

40 Jhaveri MA, Mawad HW, Thornton AC, Mullen NW, Greenberg RN. Tenofovir-associated severe bone pain: i cannot walk!. J Int Assoc Physicians AIDS Care. 2010 Sep-Oct;9(5): 328-34.

41 Mukaiyama K, Kamimura M, Uchiyama S, Ikegami S, Nakamura Y, Kato H. Elevation of serum alkaline phosphatase (ALP) level in postmenopausal women is caused by high bone turnover. Aging Clin Exp Res. 2015 Aug; 27(4):413-8.

42 Mateo L, Holgado S, Mariñoso ML, PérezAndrés R, Bonjoch A, Romeu J, et al. Hypophosphatemic osteomalacia induced by tenofovir in HIV-infected patients. Clin Rheumatol. 2016 May;35(5):1271-9.

43 Trinh S, Le AK, Chang ET, Hoang J, Jeong D, Chung $\mathrm{M}$, et al. Changes in renal function in patients with chronic HBV infection treated with tenofovir disoproxil fumarate vs entecavir. Clin Gastroenterol Hepatol. 2019 Apr; 17(5):948-56.e1.

44 Lopes JA, Jorge S. The RIFLE and AKIN classifications for acute kidney injury: a critical and comprehensive review. Clin Kidney J. 2013 Feb;6(1):8-14.

45 Lok AS, Trinh H, Carosi G, Akarca US, Gadano A, Habersetzer F, et al. Efficacy of entecavir with or without tenofovir disoproxil fumarate for nucleos $(\mathrm{t})$ ide-naive patients with chronic hepatitis B. Gastroenterology. 2012 Sep; 143(3):619-28 e1.

46 Agarwal K, Brunetto M, Seto WK, Lim YS, Fung S, Marcellin P, et al. 96 weeks treatment of tenofovir alafenamide vs. tenofovir disoproxil fumarate for hepatitis B virus infection. J Hepatol. 2018 Apr;68(4):672-81.

47 Choi J, Kim HJ, Lee J, Cho S, Ko MJ, Lim YS Risk of hepatocellular carcinoma in patients treated with entecavir vs tenofovir for chronic hepatitis B: a Korean Nationwide Cohort Study. JAMA Oncol. 2019 Jan 1;5(1):30-6.

48 Lee SW, Kwon JH, Lee HL, Yoo SH, Nam HC, Sung PS, et al. Comparison of tenofovir and entecavir on the risk of hepatocellular carcinoma and mortality in treatment-naive patients with chronic hepatitis B in Korea: a large-scale, propensity score analysis. Gut. 2020 Jul;69(7):1301-8 